\title{
Pour une éthique du discours : prise de position et rationalité axiologique
}

Arguing on behalf of an Ethics of Discourse: Taking a Stand and Axiological Rationality

\section{Roselyne Koren}

\section{(2) OpenEdition}

Journals

Édition électronique

URL : http://journals.openedition.org/aad/263

DOI : $10.4000 /$ aad. 263

ISSN : 1565-8961

Éditeur

Université de Tel-Aviv

Référence électronique

Roselyne Koren, « Pour une éthique du discours : prise de position et rationalité axiologique »,

Argumentation et Analyse du Discours [En ligne], 1 | 2008, mis en ligne le 18 septembre 2008, consulté le 10 décembre 2020. URL : http://journals.openedition.org/aad/263 ; DOI : https://doi.org/10.4000/ aad.263

Ce document a été généré automatiquement le 10 décembre 2020.

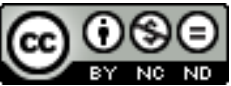

Argumentation \& analyse du discours est mis à disposition selon les termes de la licence Creative Commons Attribution - Pas d'Utilisation Commerciale - Pas de Modification 4.0 International. 


\title{
Pour une éthique du discours : prise de position et rationalité axiologique
}

\author{
Arguing on behalf of an Ethics of Discourse: Taking a Stand and Axiological \\ Rationality
}

Roselyne Koren

\section{Introduction}

1 Le chercheur en analyse du discours est nécessairement amené à problématiser des objets de recherche qu'il partage avec d'autres disciplines faisant partie des sciences humaines ou sociales. Le discours, mode de vie sociale et de régulation de l'intersubjectivité, rend ces rencontres inéluctables. Patrick Charaudeau souligne ici même ${ }^{1}$ la pertinence et le bénéfice de références à la psychologie sociale et à la sociologie quant à la définition des notions d'" influence » et de "force de l'argument ». Je souhaite pour ma part situer cet article à la croisée de l'AD, de la rhétorique argumentative et du concept philosophique d'éthique afin de problématiser la question du « positionnement » discursif.

2 Je tenterai de réexaminer cette notion au prisme du concept argumentatif de «jugement» - et par «jugement " j'entends prise de position discursive - afin de contribuer à la conceptualisation de la notion d'éthique du discours. Cette démarche a pour fin de répondre à des questions épistémologiques comme: Pourquoi la prise de parole dans l'espace public implique-t-elle que le locuteur recoure, dans de nombreux genres de discours et même si les contraintes du genre ne l'exigent pas, à l'effacement énonciatif et donne à ses opinions les apparences du jugement de fait ? Pourquoi est-il encore nécessaire aujourd'hui de rappeler que le lexique de la langue comprend des masses de termes subjectifs axiologiques et que des questions comme "Que pense X de cette question? Est-il pour ou contre ? Est-ce bien ou mal à ses yeux ? » constituent des réactions spontanées inéluctables aux dires des autres ${ }^{2}$ ? Pourquoi la majorité des 
chercheurs en sciences du langage prônent-ils un axiome déontologique de nonintervention, présupposant par là qu'elle est linguistiquement et discursivement possible, alors qu'ils reconnaissent par ailleurs la subjectivité énonciative du langage comme un fait avéré ? Pourquoi, enfin, un analyste du discours que préoccupe, certes, la question de la " véridiction ", mais tout autant celle de la " rectitude éthique " $^{3}$ et de la rationalité axiologique, se trouve-t-il contraint de recourir à une théorie de l'argumentation pour pouvoir aborder les tenants et aboutissants de l'acte de juger hors de contextes polémiques aporétiques, alors que son objet de recherche est la mise en mots et la mise en scène discursives sous toutes leurs formes?

3 Mon hypothèse est qu'il existe toujours et encore un préjugé défavorable, profondément ancré dans les doxa rationalistes du sens commun et de la recherche en sciences humaines, à l'égard de l'acte de juger et de l'argumentation conçue comme une éthique du discours ou une logique des valeurs. Ce préjugé serait dû à la confusion entre positionnement idéologique partial ou militant, parole péremptoire de donneur de leçons et acte de «jugement » argumentatif. Les sciences du langage en général et l'AD en particulier ne croient plus dans la conception d'un langage spéculaire et transparent qui pourrait dire les choses telles qu'elles sont et pourtant ce qui fascine les analystes, ce sont les mises en scène discursives qui visent à créer des effets d'objectivité et systématisent le recours à l'effacement énonciatif. Ce qui est au centre du débat ${ }^{4}$, c'est donc toujours et encore la question du rapport des dires du locuteur, ou des énonciateurs intégrés dans la trame polyphonique de ses mises en mots, à la vérité et cela, même si cette question est considérablement transformée par la notion de mise en scène subjective d'une conception socio-historique du Vrai. L'existence de la prise en charge énonciative des valeurs n'est certes pas contestée, mais elle constitue un aspect marginal, volontiers occulté, de la réflexion sur le langage et le discours. Je voudrais au contraire insister sur la nécessité de penser la coexistence et les interactions de la « rectitude éthique » et de la « véridiction ».

\section{De quelques concepts clés}

4 C'est à la croisée des concepts d'« éthique », de "sujet du discours », d'« objectivité discursive ", de "jugement de valeur» et de "responsabilité» énonciative et argumentative que se situe ma tentative de conceptualisation de la notion d'éthique du discours. Je ne prétends pas épuiser la question, ni être en mesure de proposer des réponses exhaustives à une problématique aussi complexe, mais simplement contribuer au balisage d'un terrain où trop peu de chercheurs en sciences du langage souhaitent s'aventurer.

\subsection{Brève définition opératoire du concept d'éthique}

5 Le Dictionnaire de l'Analyse du Discours publié sous la direction de Charaudeau et Maingueneau (2002), ne contient pas d'entrée «Éthique » en dépit du fait qu'il définit des concepts comme "sujet», "identité ", "ethos», " argumentation», "valeur", "modalisation» et "prise en charge» de la "vérité » ou des dires d'énonciateurs intégrés dans la trame polyphonique du discours du locuteur. On y trouve aussi une entrée «positionnement». La notion y est présentée comme "l'une des catégories de base » de l'AD, qui touche à l'instauration et au maintien d'une «identité énonciative». 
Cependant une distinction est établie entre des identités à forte "consistance doctrinale ", soit des positionnements idéologiques (" discours du parti communiste ", par exemple), et des identités "à faible consistance doctrinale ». Ainsi par exemple, pour Charaudeau, le «positionnement" correspond «à la position qu'occupe un locuteur dans un champ de discussion » et « aux valeurs qu'il défend (consciemment ou inconsciemment) et qui caractérisent en retour son identité sociale et idéologique " (2002: 453). C'est donc au prisme de concepts comme ceux de "doctrine», de militantisme ou de «normes de comportement social», "plus ou moins » choisies et assumées consciemment et librement par les sujets sociaux, que la prise de position est pensée. Il n'y est pas question de l'acte de juger et de trancher, ni de responsabilité individuelle ou collective quant aux conséquences éventuelles de ce même positionnement discursif. La question de l'éthique du chercheur, par ailleurs, n'y est pas non plus problématisée. Sans doute est-ce parce que le devoir de non-intervention est perçu comme un axiome irréfutable. Mais est-il vraiment impossible d'allier « souci éthique » et rationalité scientifique?

6 Il ne s'agira pas ici de mettre le principe de neutralité en question, mais de proposer des réajustements inspirés par une conception argumentative de l'ethos du chercheur. Je tenterai plus loin de justifier l'hypothèse qu'il pourrait exister, entre l'ethos de l'observateur impartial et celui du donneur de leçons ou du militant, une place pour l' ethos d'un chercheur qui souhaiterait énoncer, si nécessaire, des jugements de valeur raisonnés, toujours conçus a priori comme réfutables.

7 Le texte de référence dont s'inspire la définition opératoire annoncée ci-dessus est l'entrée "éthique » du dictionnaire des Notions philosophiques, publié sous la direction de Sylvain Auroux. Le concept de "sujet » y occupe une place centrale. Il y est certes présenté au prisme de l'opposition « individu \milieu » (1990: 875), du contexte social dans lequel il évolue et des contraintes qui pèsent donc sur son autonomie (1990: 870 ; 2251-2252), mais ceci ne fait pas de lui un être entièrement asservi à des injonctions doxiques. C'est la liberté, même partielle, du sujet, qui fait de lui un être autonome et moralement responsable (ibid.: 2250, 2253). Ce qui «fascine» ce sujet, ce ne sont pas uniquement les "évidences" rationalistes consensuelles (ibid.: 875), mais l'option d'une logique des valeurs qui permette de rationaliser les prises de position subjectives (ibid.: 871). Ce questionnement a la « rectitude éthique" pour objet, autrement dit le juste et l'injuste, le bien et le mal, et non pas uniquement la "véridiction ». Rationalité et jugement de valeur subjectif y sont considérés comme parfaitement compatibles. Il n'y est pas question de « comportements » sociaux ni de principes de morale extérieurs au sujet, mais de questions existentielles, de responsabilité individuelle et d'autonomie partielle, certes, mais inéluctable.

8 Le sujet éthique affronte des dilemmes qu'il lui faut résoudre en effectuant des choix, choix qui l'engagent (ibid. : 870-871; 877) en tant qu'individu et qui doivent le mener à l'action. Si la morale est constituée par un système de principes imposé de l'extérieur à une collectivité sociale, le questionnement éthique est inhérent au sujet, il "colle » à chaque individualité et correspond à une exigence intérieure de «fonder la conduite humaine, de donner un sens à la vie » (ibid. : 875). L'éthique serait un mode de réflexion individuel sur l'agir humain ${ }^{5}$.

Lesquestionnement éthique serait lié par ailleurs aux interactions verbales par des liens essentiels. La discussion contradictoire intersubjective (ibid.: 871) y est considérée comme la condition préalable, et incontournable, de la quête du juste, du 
vrai et du valide. L'éthique privilégie «l'action et, plus profondément, le langage »; il existerait des liens épistémologiques étroits entre les travaux de recherche de la philosophie analytique anglo-saxonne sur les interactions verbales et l'acception moderne du concept d'éthique, affirme le dictionnaire. La linguistique en général et l'analyse du discours en particulier laissent cependant cet espace interdisciplinaire le plus souvent inoccupé.

\section{2. «Sujet » et « prise en charge »}

Le questionnement éthique accorde une place primordiale au concept de sujet perçu comme un être à la fois autonome et soumis à des contraintes, confronté à des dilemmes existentiels et responsable de ses choix. Le sujet de l'éthique se doit d'assumer la responsabilité de la véracité de ses dires, mais il est tout autant responsable de leur intégrité. Le vrai et le juste sont à ses yeux des visées concomitantes, aussi cruciales l'une que l'autre. Je voudrais donc abonder ici dans le sens d'E. Eggs (1999: 43) ${ }^{6}$ et prendre position non pas en faveur de la primauté de la prise en charge du Vrai ou du Juste, mais en faveur d'un troisième point de vue qui insiste sur la nécessité de leur coexistence.

11 La notion de sujet joue, certes, également un rôle central dans l'analyse du discours, mais ce rôle y est très différent. Ce développement n'a pas pour fin de contester la pertinence de l'approche discursive de la notion de sujet, mais de définir ce que l'intégration d'une conception argumentative de cette même notion pourrait lui apporter quant à la question de la prise en charge. Ce dont le sujet du discours est essentiellement responsable, c'est de la mise en mots et de la mise en scène du Vrai ${ }^{7}$. Son rapport aux valeurs est celui d'un membre d'une collectivité avant d'être celui d'un individu. Son rôle se limite, dans la majorité des descriptions théoriques, à choisir, affirme Maingueneau (1999: 79), dans un ensemble préalable de «représentations sociales valorisées ou dévalorisées", celles qu'il souhaite «conforter» ou "transformer", mais la liberté dont il dispose quant à la transformation de ces représentations est constamment relativisée. Elle consiste davantage dans le choix d'un genre de discours et donc dans l'acceptation globale de ses normes et de ses stratégies que dans l'argumentation individuelle autonome de valeurs assumées explicitement.

12 Le concept de «sujet » constitue le dernier des cinq points présentés par Dominique Maingueneau (2002: 202) comme caractéristiques des «tendances françaises en analyse du discours». C'est une instance "qui à la fois se pose comme source des repérages personnels, temporels, spatiaux » et indique quelle "attitude» elle adopte à l'égard de ce qu'elle dit et de son interlocuteur. "La réflexion sur les formes de subjectivité que suppose le discours est - poursuit Maingueneau (DictAD 2002 : 189) - un des grands axes de l'analyse du discours ». L'énonciation, entendue comme acte de parole subjectif, identitaire et modalisateur, diffère donc considérablement d'une conception de l'argumentation qui renvoie à des procédures de prise de position axiologiques. Il faut de plus, lit-on dans l'entrée «Identité » (2002: 299), joindre " altérité » à "sujet ", si l'on souhaite pouvoir " utiliser la notion d'identité en analyse du discours ». "C'est à la mesure de la différence entre "soi" et "l'autre" que se constitue le sujet» (ibid.). Le "positionnement $"^{9}$ discursif de l'Un ne pourrait aboutir sans un affrontement dialogique réel ou imaginaire avec le point de vue de l'Autre. Cet Autre n'est cependant pas un alter ego, mais - affirme Maingueneau (1999: 80) - un « anti- 
garant » dont le rôle instrumental consiste avant tout à légitimer ou à valoriser les dires de l'Un.

L'ordre «contrainte» / «individuation» est inverse dans le cas du sujet de l'argumentation ou "personne» dans les termes de Perelman et Olbrechts-Tyteca (1970 : 78). La liberté y est première sans être pour autant absolue. Comment le seraitelle? Le " point de vue » du sujet qui argumente ne peut en aucun cas être celui de "Sirius », affirment Perelman et Olbrechts-Tyteca (1970: 79); il fait partie d'une collectivité évoluant dans un contexte socio-historique (ibid. : 439-440) dont il ne peut aisément enfreindre les règles. Son point de vue ne peut être qualifié de fiable et crédible sans l'accord de l'opposant; il sait et admet enfin que tout ce qui est discuté est systématiquement discutable. L'un et l'autre partagent la même «liberté de jugement " (ibid.: 73). L'allocutaire peut parfaitement refuser d'adhérer à la thèse proposée ; son autonomie est inhérente au système de l'argumentation ${ }^{10}$. L'opposant et le proposant d'une argumentation ont le même droit de choisir les opinions et les valeurs qu'ils ont à prendre en charge. La "personne " perelmanienne doit donc être considérée avant tout comme "l'auteur d'une série d'actes et de jugements", comme "un sujet libre" dont la liberté consiste dans le "pouvoir de changer et de se transformer", d'« être persuadé et de résister à la persuasion" (1970: 397). Les protagonistes d'une argumentation sont certes responsables de la vérité de leurs dires, mais ce qu'ils doivent essentiellement justifier, c'est la validité des valeurs qui régulent leurs prises de position car « le jugement permet » aussi « de juger le juge » (ibid. : 401). Celui qui prend position s'expose de ce fait à la contre-argumentation et aux risques de la disqualification... Ce qui donne du sens à l'exercice de cette liberté et le rend responsable et rationnel, c'est l'obligation dans laquelle se trouve le sujet de justifier ses actes, ses choix et ses décisions et donc de les faire comprendre aux autres tout en recourant à des "moyens discursifs" qui ont pour fin d'agir sur "l'esprit des auditeurs» (ibid.: 62). "Seule l'existence d'une argumentation, qui ne soit ni contraignante ni arbitraire, accorde un sens à la liberté humaine, condition d'exercice d'un choix raisonnable", concluent les auteurs du Traité (1970: 682). Le sujet de l'argumentation n'est donc pas un être polémique qui aurait pour fin première de "rejeter $~^{11}$ le discours de l'Autre et de lui imposer sa mise en scène du Vrai, mais l'un des protagonistes d'une négociation rationnelle qui a des prises de position axiologiques ${ }^{12}$ pour enjeu.

\subsection{Objectivité discursive et prise de position}

Le fait d'accorder la primauté à la prise en charge du Vrai conduit le locuteur à accorder une place centrale aux apparences d'objectivité et à arborer une posture réservée dès qu'il est question de prise de position. C'est au prisme de la notion de «jugement de fait » que la nouvelle rhétorique perelmanienne explore les tenants et aboutissants $d u$ "jugement de valeur $»^{13}$. Sa contribution ne se limite pas en l'occurrence à la constitution d'une somme de techniques argumentatives, elle permet de penser, dans le cas d'un discours comme celui de la presse écrite, la tension entre l'axiome de la neutralité de l'information et le concept de contre-pouvoir politique. L'argumentation peut servir de cadre théorique, à rebours des idées reçues sur la primauté de l'impartialité, à l'examen de textes et de discours où les procédures à problématiser sont, paradoxalement, celles qui affichent des apparences de neutralité. 
Perelman et Olbrechts-Tyteca consacrent le quatorzième point des «cadres de l'argumentation » à la question de la prise de position et de l'» engagement ». Il y est question de la définition du rôle du tiers, invité à servir d'arbitre en cas de conflit politique. Il s'agit donc d'un genre de discours particulier nécessairement inscrit dans un contexte socio-historique, crucial pour l'interprétation de négociations. Ce texte contient néanmoins des mises au point sur la question de l'objectivité discursive qui pourraient être utiles à tout analyste qui souhaite problématiser la question de la tension entre devoir d'objectivité et prise de position critique. Il s'agit pour les auteurs du Traité (1970 : 78) de justifier l'axiome suivant : «l'objectivité, quand elle concerne l'argumentation, doit être repensée, réinterprétée, pour qu'elle puisse avoir un sens dans une conception qui se refuse à séparer une affirmation, de la personne de celui qui la pose ». Ce qu'ils refusent d'emblée, c'est la thèse de l'existence d'un langage purement descriptif et informationnel, énoncé par un locuteur absent de son propre texte. Qui dit " argumentation » dit nécessairement pratique d'un langage géré par un sujet d'énonciation, garant et responsable de la validité de ses dires et de ses interprétations. Le but poursuivi: inciter l'Autre à adhérer aux thèses défenduesimplique que le « juge » soit capable de dissocier principe d'» impartialité » et principe d'»objectivité ». La notion d'objectivité serait fondamentalement une notion philosophique; elle ne pourrait donc être invoquée que dans un espace de discussion théorique, abstrait et décontextualisé, qui n'a rien de commun avec les " préoccupations d'ordre pratique » qui sont celles de l'argumentation et de l'action. Il serait contraire à une éthique du discours de «se placer du point de vue de Sirius » et de se retrancher dans l'abstention objective lorsque les enjeux de la prise de position sont des enjeux sociaux et qu' " une opinion exerce une influence sur l'action» (79). Le juge ${ }^{14}$ peut être « impartial » au stade heuristique de l'analyse de tous les points de vue (ou, dans les termes du discours journalistique, de l'investigation), mais il serait inadéquat qu'il adopte une posture « objective » de désengagement au moment où l'on attend de lui qu'il tranche et qu'il se prononce en faveur de l'une des positions qui lui est apparue non pas comme la seule position vraie, mais comme la solution la plus juste et la plus proche des intérêts communs des collectivités qui s'affrontent. Le tiers « objectif » ne peut évaluer les tenants et aboutissants du conflit ni contribuer à leur interprétation, car être " objectif », c'est rester extérieur, ne manifester aucune forme de « solidarité » axiologique. Ce type de distanciation est donc en contradiction avec la conception argumentative de la décision, qui implique que le juge tranche à l'intérieur de l'espace du débat. Seul le médiateur «solidaire ", qui « fait partie d'un même groupe que ceux que l'on juge, sans avoir pris préalablement parti pour aucun d'eux " pourra veiller à " un équilibre des forces » et accorder « une attention maximum aux intérêts en cause", également "répartie entre les points de vue"; seul ce médiateur « impartial », mais «solidaire » pourra permettre à l'auditoire qui le regarde faire de juger à son tour en connaissance de cause. L'argumentation permet de penser le choix responsable là où un sens commun objectiviste favorise l'option du désengagement. Ce que la nouvelle rhétorique permet donc de penser ici, ce sont les conséquences éthiques de l'effacement énonciatif, entendu comme choix de stratégies discursives de renoncement à la prise en charge de la vérité des dires des énonciateurs ou de leurs valeurs. 


\subsection{Jugement de valeur, justification et rectitude éthique} conceptualisée. Le premier exprimerait des "propositions vraies ou fausses» (ibid.: 197), le second " des attitudes propres à un individu ou à un groupe ", il peut être " plus ou moins fondé ou justifié, mais il n'est ni vrai ni faux, et ne peut donc devenir un élément constitutif d'une connaissance objective»(ibid.). Ceci implique, dans une perspective rationaliste, que l'évaluation axiologique se trouve hors du champ du rationnel et du raisonnable. Il ne reste plus, conclut Perelman, qu'à «abandonner à l'irrationnel le règlement de notre conduite ». D'où la nécessité d'une théorie de l'argumentation ou « logique des valeurs » et du "préférable » qui fonde la possibilité d'une « raison pratique » (ibid. : 198) servant à « autre chose qu'à découvrir la vérité ou l'erreur » (ibid. : 199). La garantie de la légitimité et de la validité du jugement n'est plus alors la prise en charge du discours sur le Vrai par un locuteur spéculaire impartial, mais la justification, dont la visée serait «pratique» (ibid.: 198). Celle-ci «concerne essentiellement une action ou une disposition à agir : on justifie un choix, une décision, une prétention » (ibid. : 198) et ce faisant, on les rattache á «l'idée de valorisation ou de dévalorisation" (ibid.: 199). Le locuteur prend l'énonciation et son adhésion à une thèse explicitement en charge tout en admettant $a$ priori la légitimité de la réfutation et le faitque la justification concerne «ce qui est à la fois discutable et discuté " (ibid.: 198). L'argumentateur montre en justifiant ses dires qu'il reconnait d'emblée que ce qu'il argumente n'est pas une évidence " inconditionnellement et absolument valable ", mais une prise de position discutable, raisonnée et subjective. "Doit être justifié " «pour un esprit épris de rationalité, ce qui n'est ni évident ni arbitraire » (ibid.: 201) : l'argumentation refuse le caractère réaliste absolu du raisonnement rationaliste et étend la rationalité au champ de l'évaluation affective et/ou axiologique où rien n'est « évident » et où tout peut aisément être perçu comme discutable.

Ce qui est en question ici, ce n'est pas seulement l'acte de parole par lequel celui qui justifie ses prises de position tente de se soustraire à tout "reproche» et à tout "blâme ", mais aussi un type de rapport différent au savoir. La justification peut en effet concerner également « une disposition à croire, une prétention à savoir » (ibid. : 206). Il devrait donc y avoir, aux côtés d'une théorie cognitive rationaliste, qui articule «droit à la certitude » et exigence de vérité, et en interaction avec elle, une théorie de l'argumentation qui propose des "règles méthodologiques" et conduit à des «considérations d'ordre épistémologique». Le refus de "limiter le rationnel à l'évident ", la décision de défendre une approche axiologique du jugement constituent la condition qui permette de fonder une axiologie rationnelle « de l'action et de la pensée » (ibid. : 206) et une "théorie de la "connaissance" " intégrant une logique des valeurs. La nouvelle rhétorique perelmanienne ne nie pas la pertinence ni la nécessité d'une conception rationaliste du savoir. Ce qu'elle conteste, c'est la limitation de la 
définition des normes du discours argumentatif valide à la véridiction ou mode de transmission correcte de la vérité des prémisses à la conclusion, défini par Plantin (2002: 72). Elle permet de ce fait de penser des questions comme la «rectitude éthique ", la responsabilité du locuteur et la «mauvaise foi argumentative $»^{15}$ - et par rectitude éthique, j'entends : devoir de justification raisonnée du point de vue, mise en mots explicite de la visée de l'argumentation, identité et responsabilité énonciatives assumées explicitement, attribution à l'allocutaire du rôle crucial de juge de la pertinence et de la validité des dires de l'Un, respect de sa liberté de pensée ${ }^{16}$.

\subsection{De la responsabilité discursive à la responsabilité argumentative}

Le terme "responsabilité " n'apparaît pas dans les entrées "Argumentation", " Autorité », «Acte de langage ", "Acte de langage indirect ", "Acteur ", " Analyse du discours ", "Ethos ", «Individuation", «Subjectivité " du Dictionnaire d'Analyse du Discours, mais il y fait quelques apparitions ponctuelles dans les entrées "Actant», "Action langagière ", «Énonciateur ", « Énonciation » et "Sujet du discours ». Il y est précisé par ailleurs qu'»Action langagière" ne serait pas "une entité d'ordre linguistique »; cette notion envisage le concept de " responsabilité » « dans le cadre de "l'interactionnisme socio-discursif" ", d'un " point vue psychologique » (2002:25). Il y est question de la responsabilité attachée à " la subjectivité parlante » de l'énonciateur qui accomplit des «actes de langage » et de celle du «locuteur », au sens ducrotien du terme, seul « responsable de l'énonciation » $(2002: 224)$. Le terme « responsable » n'est d'ailleurs utilisé dans la définition des acceptions de «sujet du discours » que dans le cas du " locuteur » d'Oswald Ducrot, « être de discours » opposé à « un être empirique ", " extérieur à tout acte de langage » (2002: 554). Il s'agirait essentiellement en fait d'un type de responsabilité « juridique $»^{17}(2002: 225)$.

On trouve également sous la plume de Maingueneau (1998: 41) le terme d'énonciateur «responsable» entendu comme "garant de la vérité de l'énoncé». Ce type de responsabilité vériconditionnelle est en fait comparable aux conditions d'acceptabilité du «point de vue logico-scientifique » défini dans l'entrée "Autorité » du Dictionnaire d'Analyse du Discours (2002: 85). On peut y lire en effet qu' " un discours est recevable s'il recueille et articule selon des procédures admises des propositions vraies, c'est-à-dire conformes à la réalité $»^{18}$. Ce qui n'est donc pas problématisé, c'est la description d'un autre type d'«engagement» discursif qui ne concerne pas la vérité du contenu propositionnel, mais l'énonciation et l'argumentation de jugements de valeur à fins persuasives, dont l'énonciateur serait un sujet conçu comme « une force agissante » et " une cause $~^{19}$ et non pas comme un sujet » surdéterminé ", « contraint ", « dominé $»^{20}$. Les locuteurs d'une langue ne sont pas des être désincarnés qui se contentent de prendre la parole en cas de litige sur des questions de vérité référentielle, ni des entités libérées par des instances juridiques du devoir d'introspection et de réflexion qui a la "conscience intérieure de soi ${ }^{21}$ pour fondement. Le recours aux constituants axiologiques du lexique et des pratiques discursives serait-il dispensé de toute forme de responsabilité énonciative ? Et pourquoi faudrait-il que le linguiste renonce à penser ce qui sort du cadre de la prise en charge de la vérité référentielle ou des actes d'énonciation polyphoniques? Les linguistiques de l'énonciation et l'analyse du discours attribuent une place centrale, dans leurs prises de position théoriques, à la 
subjectivité et à l'intersubjectivité. Il est donc surprenant que cela ne mène qu'à une réflexion partielle sur la responsabilité discursive d'un énonciateur qui est aussi amené à prendre position en termes axiologiques, termes qui impliquent nécessairement un appel à l'Autre et la volonté ou la tentative d'exercer une influence sur autrui.

Ducrot est l'un des rares linguistes qui problématisent la question de la responsabilité énonciative envers la prise en charge du Vrai. Mais il s'agit en fait d'un type d'approche négatif qui prend pour objet les stratégies discursives de dénégation ou de masquage des prises de position du sujet. Ainsi met-il essentiellement l'accent sur la « difficile liberté » d'un locuteur occupé à résister aux contraintes de la doxa ou aux assauts d'interlocuteurs qui exploiteraient l'intentionnalité inhérente à toute prise de parole pour exercer un droit de regard sur ses dires ${ }^{22}$. L'éventualité de la contreargumentation constituerait une menace pour l'ethos du sujet d'énonciation, contraint d'assumer la responsabilité de ses dires et de les justifier explicitement; celui-ci pourrait par exemple résoudre la difficulté en donnant à sa prise de position l'apparence d'un énoncé comme « A donc C » qui arbore les apparences du jugement de fait et l'autorité d'une logique déductive rationaliste. Il accomplit, ce faisant, affirme Ducrot (2004 : 26), un « coup de force " polémique destiné à réduire son interlocuteur au silence. L'énonciateur serait contraint s'il souhaite aller à rebours des idées reçues, des tabous et des interdits énonciatifs "protégés par une sorte de loi du silence ", de recourir à des stratégies de masquage identitaire qui lui permettraient de «dire sans encourir la responsabilité d'avoir dit » et donc sans avoir à rendre compte de ses dires (1972: 4-6).

22 Contrairement à la théorie ducrotienne de l'argumentation dans la langue, l'«argumentation rhétorique » perelmanienne met l'accent sur l'autonomie et la responsabilité du sujet d'énonciation qui ne souhaite pas s'effacer ni « dire et ne pas dire ", mais au contraire prendre position et trancher. Perelman critique la «tradition scientifique et philosophique occidentale $»^{23}$ qui alimenterait le mythe de la parole autonome des faits et validerait ainsi la transformation du sujet responsable en un «trou d'être » ou "néant» qui ne ferait pas écran entre «l'idée " et l'« objet de la connaissance ${ }^{24}$. Le sujet d'énonciation est certes à ses yeux un "être social» (j'emprunte ce terme à Charaudeau 2005 : 232), mais il n'en est pas moins un individu autonome et contraint, du fait de cette autonomie, de trancher et de prendre position. La conception rhétorique de l'argumentation ne présente pas la responsabilité énonciative comme un joug ni le «jugement du je» (Pottier 1992: 76) comme un délit ou un risque "redoutable »; elle ne distingue pas sur ce point entre le chercheur (j'y reviendrai) et les autres « êtres sociaux »...

Dernier point particulièrement éclairant quant aux liens qui lient la question de la responsabilité énonciative à celle de l'autonomie du sujet du discours : la distinction entre moralisation et questionnement éthique. Perelman (1945:9) distingue entre argumenter, soit confronter et justifier rationnellement des choix éthiques contraires ${ }^{25}$, et « moraliser » entendu comme tirer profit de « mots à résonance émotive » ou " mots que l'on écrit avec une majuscule pour bien montrer le respect qu'on leur témoigne »: Justice, Liberté, Beau, Devoir, etc. Ce qu'il considère comme condamnable, c'est l'exploitation du prestige de ces valeurs pour tenter d'imposer par la force des prises de position idéologiques. Si les censeurs et les donneurs de leçons ne sont pas en odeur de sainteté et si l'acte de juger est considéré comme suspect, c'est en raison d'une des "deux composantes » qui constituent la «notion confuse de morale » : le "moralisme » 
ou, affirme Marc Dominicy (2002: 135), « qualité apparente des esprits faibles, enclins à l'obéissance aveugle et soucieux de se conformer à l'opinion dominante » et la morale authentique, qui serait « la qualité réelle de ceux qui restent capables d'évaluer leurs actes en termes éthiques, au delà des modes ou pressions du moment ». La moralisation impliquerait un comportement autoritaire qui aurait surtout la responsabilité de l'Autre pour cible. Elle se reconnaîtrait à la «fascination " pour les «évidences » de «l'ordre établi » et à « un monde de normes » où il n'y aurait pas de place pour le choix individuel ${ }^{26}$. Or le questionnement éthique problématise la responsabilité de tous les protagonistes de l'interaction verbale, il "colle à chaque individualité » et implique « une exigence de renouvellement » et d'» inventivité personnelle ».

\section{De la théorie à la pratique}

Je me propose à présent de justifier les prises de position épistémologiques défendues dans la première partie de cet article en proposant trois études de cas. Les deux premières (2. 1 et 2.2$)$ concernent l'éthique du discours journalistique. La troisième (2. 3), celle du discours scientifique. Les analystes de la nouvelle rhétorique soulignent dans la plupart des cas les liens qui lient cette théorie de l'argumentation aux discours philosophique et/ou juridique, mais Perelman et Olbrechts-Tyteca affirment dès le début du Traité:

Nous chercherons à la construire [la théorie de l'argumentation] en analysant les moyens de preuve dont se servent les sciences humaines, le droit et la philosophie, nous examinerons des argumentations présentées par des publicistes dans leurs journaux, par des politiciens dans leurs discours, par des avocats dans leurs plaidoiries, par des juges dans leurs attendus, par des philosophes dans leurs traités (1970: 13).

\subsection{Le cas de l' " engagement neutre ${ }^{27}$ de l'écriture de presse : «Evidences»}

Je me propose à présent de montrer, en commentant trois extraits d'un article du Monde (18 avril 2003) intitulé «Evidences", ce qu'une approche argumentative du concept d'« engagement neutre » permet de problématiser et d'éclairer. Ces extraits, les voici ${ }^{28}$ :

1. Dans le tumulte, le désordre, l'anarchie, l'insécurité, la pénurie, bref, dans tout ce qui fait le quotidien d'un pays, et surtout de ses villes dévastées par la guerre, au moins une vérité apparaît, jour après jour, au travers des découvertes et des témoignages. C'était une évidence déjà c'est un fait établi désormais : le régime de Saddam Hussein fut bien cette abominable dictature, imposant par la terreur, la répression, la torture et les exécutions son propre pouvoir et sa propre longévité.

2. Il faudrait donc être d'une assez détestable mauvaise foi pour ne pas reconnaître que d'un mal, la guerre, est sorti un bien, sans aucun doute très fragile et précaire, la fin d'une oppression abominable.

3. D'où peut-être la nécessité, en guise d'alibi à ses propres accommodements avec une situation connue et délibérément occultée pour des intérêts supérieurs, de trouver un prétexte à l'action après des décennies d'inaction. Ce fut, cette fois, la recherche d'armes de destruction massive. En trouvera-t-on? Ou pas? La question reste ouverte. La réponse, elle, fut fermée et définitive.

Il ne s'agit pas ici d'imputer au journaliste la responsabilité de l'oscillation et de l'interaction, inhérentes au système de la langue, entre structures linguistiques et 
discursives contraignantes et autonomie, mais de montrer comment le journaliste met en pratique et régule ce système dans un article à visée argumentative auquel la rédaction a donné le titre paradoxal d' "Evidences " ${ }^{29}$. L'article se situe d'emblée dans le champ du Vrai absolu et de réalités présentées comme objectives et indiscutables. Ce qui retient l'attention, dès le titre, c'est le ton péremptoire, l'ethos autoritaire d'un médiateur qui affiche les apparences d'un rapport direct avec des faits présentés comme avérés et la tentative de faire croire que de tels faits peuvent exister dans un contexte politique (il s'agit d'un bilan critique de la seconde guerre contre l'Irak). On pourrait s'attendre à ce que la suite soit de l'ordre du compte rendu ou du procès verbal, mais il n'en est rien : on y trouve de nombreuses qualifications axiologiques ou émotionnelles comme "désordre», "pénurie ", "villes dévastées", «abominable dictature », « répression », « oppression abominable », « décennies d'inaction » etc. Il y a donc simultanément apparences objectives affichées tout au long du texte et prises de position qui impliquent des jugements de valeur. Ces deux ethè, celui du rapporteurmiroir et celui de l'analyste critique (« devoir d'irrespect» du journaliste, fonction de " contre-pouvoir ») semblent non pas se compléter ni se légitimer réciproquement, mais œuvrer côte à côte à un coup de force discursif qui aurait pour but d'intimer à l'auditoire de considérer le point de vue défendu par le journaliste comme la vérité absolue. Au lieu d'assumer explicitement ses prises de position, ce qui serait tout à fait légitime dans ce type d'article - il s'agit d'un éditorial - le journaliste accumule les effets d'objectivité et semble chercher à imposer ce qu'il aurait dû tenter de justifier et de faire accepter en argumentant son point de vue. Voici quelques-uns des énoncés où figurent ces pratiques discursives: "au moins une vérité apparaît... ", "c'était une évidence déjà c'est un fait établi désormais ", «ce fut, cette fois, la recherche d'armes de destruction massive». On y reconnaît des stratégies bien connues comme l'attribution à un concept, la "vérité", d'une vie autonome indépendante de l'interprétation humaine, l'articulation du présentatif « objectif » impersonnel « c'est » et des notions d' «évidence » et de «fait établi » qui présupposent qu'il existe a priori de telles notions dans l'espace politique international et que le rapporteur-miroir peut les refléter telles quelles, le sous-entendu dont "cette fois ", notation chronologique aux apparences anodines, est le point de départ car il implique le raisonnement implicite suivant : ce n'est pas la première fois où la grande puissance mise en cause ici recourt à un prétexte fabriqué de toutes pièces pour justifier son entrée en guerre. Les prises de position sont donc affirmées par le biais de qualifications subjectives, mais elles ne sont pas pleinement assumées. L'auteur systématise le recours à l'effacement énonciatif dans les exemples cités ci-dessus, mais aussi dans la totalité de l'article. Son rôle se limite à la médiatisation d'une voix anonyme, celle de la parole de "vérités " qui émergent d'elles-mêmes. Quant aux critiques dirigées contre des instances politiques internationales, critiques qui impliquent un second ethos antithétique (cf. plus haut les qualifications axiologiques et/ou affectives), elles pointent des responsabilités, imputent des fautes (3.: «alibis » politiques mensongers en guise de justifications, refus de voir et de savoir ce qui est contraire aux intérêts de l' État, « inaction », etc.), mais sans dire explicitement à qui. Ainsi, le texte ne précise pas aux yeux de quel État il y a «nécessité » ni quel est celui qui s'est «accommodé » et a "occulté » les savoirs dérangeants; il ne précise pas davantage qui a pris l'initiative de la «recherche d'armes » ni qui a veillé à ce que la réponse soit « fermée et définitive ».

Ce que l'approche argumentative permet donc de percevoir, ce sont les modalités de l'acte de juger, la réticence de l'auteur à assumer explicitement ses prises de position 
axiologiques en recourant à de nombreux effets d'objectivité parmi lesquels l'effacement énonciatif occupe une place de choix. Ces passages permettent d'observer les pratiques discursives d'une démarche qui consiste à dire, à savoir prendre position, énoncer des jugements de valeur, et à masquer aussitôt ce que l'on vient de dire en systématisant l'effacement énonciatif. L'euphémisation des prises de position serait une stratégie de "rachat» (j'emprunte ce terme à François Rastier) car ce qui compterait en fait, quant à la légitimation publique des dires du journaliste, ce serait les apparences du Vrai, l'accès à peine médiatisé, en direct, à des évidences préétablies. Ce qui n'aboutit pas ici, d'un point de vue argumentatif, c'est le questionnement qui doit permettre à l'auditoire de passer de la réflexion à l'action. La réflexion sur la responsabilité des États impliqués dans le conflit est remplacée par un discours de condamnation qui se contente de moraliser et de chercher des coupables. Là où il aurait fallu argumenter, problématiser la question de la responsabilité collective et des conséquences de la guerre au prisme d'une "raison pratique», soit débattre de l'avenir ${ }^{30}$, le journaliste se contente de la posture d'«imprécateur " qui recourt à l'intimidation pour avoir raison de ceux qui oseraient soutenir un point de vue différent.

La seconde citation illustre clairement ces points : « il faudrait donc être d'une assez détestable mauvaise foi pour ne pas reconnaitre que». Le ton catégorique de la tournure impersonnelle - effet d'objectivité notoire - est relativisé ici par la valeur modale du conditionnel d'éventualité, mais l'accusation de " détestable mauvaise foi » constitue une double menace - le droit au désaccord n'est pas reconnu à l'auditoire. Celui-ci n'aurait que deux options: s'aligner, ou être exclu du groupe de ceux qui détiennent le monopole de la définition des « évidences".

Contrairement au journaliste, le "proposant " d'une thèse argumentative veille à justifier des thèses qu'il ne saurait en aucun cas qualifier d' "évidences ", sans chercher pour autant à faire taire ses passions. Il fait tout ce qui est en son pouvoir pour persuader l'auditoire, en lui reconnaissant néanmoins le droit de le réfuter et de ne pas adhérer à ses thèses car il sait et admet que tout ce qui est «discuté » doit être considéré comme « discutable ».

\subsection{Le cas de l'écriture "bipolaire ${ }^{31}$ ou l'oscillation binaire au prisme d'un rationalisme " égalitaire »}

Un des rythmes fondamentaux de l'écriture de presse est assurément l'oscillation "statique $~^{32}$ entre des pôles contraires ou intrinsèquement différents. Mon intention n'est pas ici de recenser ni d'analyser les diverses causes de ce type de cadence ${ }^{33}$, mais d'établir un lien entre cette cadence fondamentale et l'un des types de rationalisme critiqué par la nouvelle rhétorique : le « rationalisme égalitaire » (Perelman 1989 : 202). Nous avons vu plus haut que la nouvelle rhétorique intègre l'impartialité dans le champ de l'éthique au stade heuristique de l'analyse des points de vue, mais qu'elle la dissocie de l'objectivité, jugée incompatible avec les nécessités de l'action, à tous les stades des procédures argumentatives qui ont une prise de position finale pour enjeu. Il serait contraire aux normes d'un rationalisme "égalitaire ", mais aussi à celles d'une théorie de l'argumentation, d'établir, dans le cadre préliminaire du recensement « impartial » des points de vue conflictuels, la moindre hiérarchie entre les diverses thèses en présence. Les deux approches se séparent néanmoins à l'étape suivante. Là où le 
partisan d'une éthique objectiviste refuse de se livrer à la moindre hiérarchisation au nom d'un idéal égalitariste, celui d'une éthique argumentative, fort proche sur ce point de l'éthique de responsabilité de Max Weber, prône une prise de position axiologique, la justification de la valorisation de l'Un et de la dévalorisation de l'Autre, la hiérarchisation et la décision. L'obligation de choisir et de prendre position joue un rôle central dans la nouvelle rhétorique où l'ultime preuve de la réussite d'un argumentaire est le passage de la pensée à l'action; l'oscillation «statique » systématique entre des options antithétiques ne saurait y être une fin en soi. Le scepticisme qui conduit à la décision de ne pas décider est aux yeux des auteurs du Traité aussi dangereux que le fanatisme. Le sceptique "refuse » l'engagement parce qu'il assimile «l'adhésion à une thèse à la reconnaissance de la vérité absolue de celle-ci ». Or l'argumentation « ne peut fournir des justifications qui tendraient à montrer qu'il n'y a pas de choix, mais qu'une seule solution s'offre à ceux qui examinent le problème ». Bref, le sceptique « se fait de l'adhésion - affirment Perelman et Olbrechts-Tyteca (1970:82-83) - une idée qui ressemble à celle du fanatique : l'un et l'autre méconnaissent que l'argumentation vise à un choix entre des possibles». La rationalité du choix n'est pas garantie par la "vérité absolue de la thèse ", mais par la justification explicite de la hiérarchisation des points de vue et d'une décision qui est liée à un « engagement » conscient et délibéré du sujet. On pourrait être tenté de voir des similitudes entre l'incertitude engendrée par l'oscillation binaire systématique et quelques-uns des leitmotiv épistémologiques de la nouvelle rhétorique comme : il n'y a pas de définition intrinsèque absolue du " bon » et du « mauvais » argument, tout argument aussi fort soit-il peut-être discuté et réfuté, la nécessaire confrontation du pour et du contre, la relativisation contextuelle du sens de concepts pourtant cruciaux aux yeux du théoricien comme "conviction" et " persuasion ", « auditoire universel » et " auditoire particulier », mais ce n'est qu'une illusion d'optique. Le caractère flou et confus des notions, leur plasticité seraient en fait aux yeux de Perelman, dans le cas de nombreux dilemmes éthiques, au service de la part d'autonomie inhérente à tout sujet d'énonciation. Ils n'alimenteraient pas les velléités de désengagement, mais encourageraient les dispositions du sujet à choisir, trancher et agir le plus librement possible dans un contexte socio-historique précis.

Le rationalisme égalitaire risque d'œuvrer au maintien du statu quo, mais aussi de constituer la condition de possibilité de raisonnements « quasi logiques » où le vrai et le faux, des vérités historiques établies et leur dénégation, la criminalisation et l'idéalisation concomitantes du même individu seraient présentés comme des thèses équivalentes ou transformés en questions de goût ${ }^{34}$. Ce qui est problématique en l'occurrence, c'est l'attitude "objective » et distanciée d'une instance médiatique qui s'exonère de la responsabilité de trancher, définie en ces termes par le philosophe Vincent Descombes dans Philosophie par gros temps :

donner une identité morale à ce qui vient d'arriver [...] dans des mots qui [...] préparent la communauté aux décisions qu'appelle cet événement. Seul un événement qui ne requiert aucune décision personnelle et collective peut se contenter d'une identité physique, d'une description donnée dans un vocabulaire neutre. Mais si le groupe auquel nous appartenons doit réagir, il faut que le changement récent ou imminent de l'état des choses lui soit annoncé dans une langue qui fixe clairement les responsabilités et les mérites, les tâches et les droits : ce qui arrive veut dire menace ou secours, victoire ou défaite, crime ou châtiment (Minuit, 1989 : 77). 


\subsection{Contribution au débat sur la neutralité du chercheur}

32 Je voudrais problématiser au moment de conclure la question de la prise de position du chercheur. L'axiome de non-intervention est profondément ancré dans la recherche en sciences humaines. La dérogation à cet impératif fait courir à qui ose l'enfreindre le risque de se voir refuser la qualification de scientifique et donc de se voir refuser par ses pairs le droit à la parole. Toute forme de jugement de valeur est aussitôt qualifiée, avec une réprobation non dissimulée ${ }^{35}$, de «militante» et/ou de "normative». Il ne s'agit pas ici de mettre la légitimité ni la pertinence de ce principe en cause ni d'argumenter en faveur de la thèse inverse qui revendiquerait pour le chercheur le statut permanent d'acteur et non d'analyste «objectif». Je voudrais simplement rappeler ici ${ }^{36}$ quelle est la position de la nouvelle rhétorique en la matière. L'AD éclaire bien la question des contraintes sociales et institutionnelles qui pèsent sur l'attribution $\mathrm{du}$ droit à la parole ainsi que la question des pratiques discursives imposées par le concept de genre, mais elle ne traite pas la question de la rectitude discursive (c'est son droit le plus légitime $)^{37}$. Le chercheur devrait donc s'en tenir à décrire par exemple l'oscillation binaire entre deux pôles contraires et renoncer à sa « liberté de jugement " là où il existe d'autres options comme celle de désigner et d'imaginer les conséquences éventuelles de ce type d'écriture «bipolaire » dans les cas où le vrai et le mensonger sont présentés comme équivalents. Je suis parfaitement consciente du fait qu'un linguiste ne peut pas évaluer les effets perlocutoires des discours ou des argumentaires qu'il analyse, pour des raisons épistémologiques, mais je suis convaincue qu'il peut en imaginer ou en calculer quelques-uns à partir de l'analyse attentive de stratégies discursives d'ores et déjà classées et répertoriées. Si l'argumentation, nous l'avons vu plus haut, peut se constituer en théorie de la connaissance, c'est, entre autres, parce qu'elle propose des outils d'analyse épistémologiques.

Ceci implique, par exemple, que dans le cas de l'assimilation polémique d'une armée contemporaine à celle de la Wehrmacht du régime nazi, je ne me placerai pas du point de vue de la réception de la procédure qui peut osciller entre l'indifférence, la légitimation ou l'indignation, mais à l'intérieur du rapprochement; j'essaierai de démontrer où se situe la procédure assimilatrice fallacieuse par rapport à des normes éthiques argumentative de «rectitude». Il existe des définitions consensuelles de l'argument par analogie, indépendantes de la situation d'énonciation, qui permettent de démontrer dans quels lieux discursifs des similitudes ponctuelles peuvent dévier et se transformer en assimilation totale avec, en l'occurrence, le parangon du Mal, à des fins de délégitimation de l'ethos de l'autre. Je rappellerai ensuite que l'« amalgame » (on trouve même, aujourd'hui, ce terme polémique dépréciatif sous la plume des plus ardents défenseurs du devoir de neutralité scientifique) peut contribuer à la légitimation implicite de la mise à mort d'un ennemi politique comme l'atteste l'histoire de l'emploi du mot dans les procès révolutionnaires où le verdict - envoyer à la guillotine les ennemis politiques de la Révolution - avait pour condition de possibilité leur « amalgame » préalable avec des détenus, coupables de crimes de droit commun.

La publication ces dernières années de travaux de recherche qui n'hésitent pas, par exemple, à analyser le fonctionnement des médias en termes critiques ni à stigmatiser les dérives et les dérapages de l'idéologie de captation qui transformerait l'information en manipulation pourrait contribuer à démontrer que le principe de non-intervention absolue est un principe intenable. La prise de position axiologique et les justifications 
qui la rationalisent constituent, si nécessaire, une option valide pour le chercheur qui souhaite, comme l'affirment les auteurs du Traité de l'argumentation (Perelman et Olbrechts-Tyteca $1970: 681-682)$, pratiquer un «rationalisme critique » et assumer la responsabilité « de ses décisions dans le domaine de la connaissance comme dans celui de l'action».

\section{BIBLIOGRAPHIE}

Adam, Jean-Michel et Bonhomme, Marc. 1997. L'argumentation publicitaire (Paris : Nathan)

Adam, Jean-Michel et Lugrin, Gilles. 2006. « Effacement énonciatif et diffraction co-textuelle de la prise en charge des énoncés dans les hyperstructures journalistiques », Semen 22 (Rabatel, Alain et Chauvin-Vileno, Andrée, éds), pp. 127-144

Amossy, Ruth (éd.). 1999. « L'ethos au carrefour des disciplines. Rhétorique, pragmatique, sociologie des champs », Images de soi dans le discours (Lausanne-Paris : Delachaux et Niestlé) Amossy, Ruth. 2006 [2000]. L'argumentation dans le discours (Paris : Armand Colin).

Auroux, Sylvain. 1990. Les Notions philosophiques, tome I-II, Encyclopédie Philosophique Universelle (Paris : PUF)

Boudon, Raymond. 1995. « Sens et Raisons. Théorie de l'argumentation et Sciences Humaines ", Hermès 16, «Argumentation et Rhétorique » (Paris : CNRS Editions), pp. 29-43

Boutet, Josiane, Gardin, Bernard et Lacoste, Michèle. 1995. « Discours en situation de travail ", Langages 117, « Les analyses du discours en France », pp. 12-31

Charaudeau, Patrick. 1992. Grammaire du sens et de l'expression (Paris : Hachette)

Charaudeau, Patrick. 1997. Le discours d'information médiatique, La construction du miroir social (Paris : Nathan)

Charaudeau, Patrick et Maingueneau, Dominique. 2002. Dictionnaire d'Analyse du Discours (Paris : Seuil)

Charaudeau, Patrick. 2005. «Quand l'argumentation n'est que visée persuasive. L'exemple du discours politique », Burger, Marcel, et Martel Guylaine (éds). Argumentation et communication dans les medias (Québec : Editions Nota Bene)

Coltier, Danièle et Dendale, Patrick. 2004. « La modalisation du discours de soi : éléments de description sémantique des expressions "pour moi", "selon moi" et à mon avis" ", Langue Française 142, pp. 41-57

Dendale, Patrick et Coltier, Danièle. 2005. « La notion de prise en charge ou de responsabilité dans la théorie scandinave de la polyphonie linguistique », Bres, Jacques, Haillet, Patrick Pierre, Mellet, Sylvie, Nølke, Henning et Rosier, Laurence (éds). Dialogisme et polyphonie. Approches linguistiques (Bruxelles : De Boecket Duculot), pp. 125-140 
Dominicy, Marc. 2002. "La dimension sémantique du discours argumentatif », Koren, Roselyne et Amossy, Ruth (éds). Après Perelman : Quelles politiques pour les nouvelles rhétoriques? (Paris :

L'Harmattan), pp.123-152

Ducrot, Oswald. 1972. Dire et ne pas dire (Paris : Hermann)

Ducrot, Oswald, 2004. « Argumentation rhétorique et argumentation linguistique », Doury, Marianne et Moirand Sophie (éds). L'argumentation aujourd'hui, (Paris : Presses Sorbonne Nouvelle), pp. 17-34

Eggs, Ekkehard. 1999. « Ethos aristotélicien, conviction et pragmatique moderne », Amossy, Ruth (éd.). Images de soi dans le discours (Lausanne : Delachaux et Niestlé)

Kerbrat-Orechionni, Catherine. 1980. L'énonciation : de la subjectivité dans le langage (Paris : Colin)

Kerbrat-Orechionni, Catherine. 1981. «Argumentation et mauvaise foi », L'argumentation (Lyon : PUL), pp. 41-63

Koren, Roselyne. 1993. « Perelman et l'objectivité discursive : le cas de l'écriture de presse en France ", Chaim Perelman et la pensée contemporaine (Bruxelles : Bruylant), pp. 469-487

Koren, Roselyne. 1996. Les enjeux éthiques de l'écriture de presse et la mise en mots du Terrorisme (Paris : L'Harmattan)

Koren, Roselyne. 2001. «Quand informer, c'est dire le blanc et le noir : quelques effets pervers de l'oscillation binaire », Boogaards, P., Roonyck, J. et Smith, J. (éds). Quitte ou Double Sens (Amsterdam : Rodopi), pp. 177-200

Koren, Roselyne. 2002. "La "Nouvelle Rhétorique“, "technique“ et/ou "éthique" du discours : le cas de l' "engagement" du chercheur ", Amossy, Ruth et Koren Roselyne (éds). Après Perelman: quelles politiques pour les nouvelles rhétoriques? L'argumentation dans les sciences du langage (Paris :L'Harmattan), pp. 197-228

Koren, Roselyne. 2003. «L'engagement de l'Un dans le regard de l'Autre : le point de vue d'une linguiste ", Questions de communication, 4, pp. 271-277

Koren, Roselyne. 2004. "Argumentation, enjeux et pratique de l' "engagement neutre“", Semen, no. 17 , Besançon, pp. 19-40

Koren, Roselyne. 2006. «Quels risques pour quelles prises de position "normatives“" ?, Questions de communication, no. 9, pp. 195-205

Koren, Roselyne. 2006a. «La responsabilité des Uns dans le regard des Autres : l'effacement énonciatif au prisme de la prise de position argumentative ", Semen, no. 22, pp. 87-94

Langages 1995, no. 117, Maingueneau, Dominique (éd.). « Les analyses du discours en France »; «Présentation », pp. 5-11

Maingueneau, Dominique. 1998. Analyser les textes de communication (Paris : Dunod)

Maingueneau, Dominique. 1999. «Ethos, scénographie, incorporation », Amossy, Ruth (éd.).

Images de soi dans le discours (Lausanne : Delachaux et Niestlé)

Moirand, Sophie. 2006. « Responsabilité et énonciation dans la presse quotidienne ; questionnements sur les observables et les catégories d'analyse », Semen, 22, pp. 45-59

Morin, Violette. 1969. L'écriture de presse. (Paris et La Haye : Mouton)

Perelman, Chaim. 1945. De la justice (Bruxelles : Editions de l'Université de Bruxelles) 
Perelman, Chaim et Olbrechts-Tyteca, Lucie. 1970 (4e éd.). Le Traité de l'argumentation. La nouvelle rhétorique (Bruxelles : Editions de l'Université de Bruxelles)

Perelman, Chaim. 1989. Rhétoriques (Editions de l'Université de Bruxelles)

Plantin, Christian. 2002. « Argumentation », « Valeur », Charaudeau, Patrick et Maingueneau, Dominique (éds). Dictionnaire de l'Analyse du Discours (Paris : Le Seuil)

Plantin, Christian. 2002 a. « Analyse et critique du discours argumentatif », Koren Roselyne et Amossy Ruth (éds). Après Perelman. Quelles politiques pour les nouvelles rhétoriques? L'argumentation dans les sciences du langage (Paris : L'Harmattan)

Pottier, Bernard. 1992. Sémantique générale (Paris : PUF)

Rabatel, Alain et Chauvin-Vileno, Andrée. 2006. «La question de la responsabilité dans l'écriture de presse », Semen, 22, pp. 7-27

Rabatel, Alain. 2006. «L'effacement de la figure de l'auteur dans la construction événementielle d'un "journal“ de campagne électorale et la question de la responsabilité en l'absence de récit primaire », Semen, 22, pp. 77-92

Rabatel, Alain (à paraître). « Prise en charge et imputabilité, ou la prise en charge à responsabilité limitée... »

Searle, John Rogers. 1972. Les actes de langage (Paris : Hermann)

Searle, John Rogers. 1982. Sens et expression, « Le statut logique du discours de la fiction » (Paris : Minuit), pp. 101-119

Rabatel, Alain et Chauvin-Vileno, Andrée (éds) 2006, « Énonciation et responsabilité dans les médias » (Semen, 22)

Tappolet, Christine. 2000. Emotions et valeurs (Paris : PUF)

\section{NOTES}

1. Voir «L'argumentation dans une problématique de l'influence » : «C'est ce que j'appelle une 'interdisciplinarité focalisée'. Ainsi en est-il, pour ce qui me concerne, de la problématique de l'influence, des concepts de communication, de représentation et d'effets que j'emprunte largement à la psychologie sociale et à la sociologie, mais que je redéfinis dans le champ langagier ».

2. Cf. Kerbrat-Orecchioni $1980: 82$, où l'auteur évoque le « besoin d'encodage qui se répercute au décodage sous la forme de ce réflexe interprétatif dont on fait constamment l'expérience : que pense celui qui parle de l'objet dont il parle ? est-il 'pour', est-il 'contre'? Besoin de réflexe dont il est plus facile de s'irriter que de se défaire ».

3. Cf. la définition de l'entrée « Argumentation » du Dictionnaire d'Analyse du Discours 2002: 72.

4. Cf., ici même, Charaudeau, art. cité : « l'analyse du discours n'a pas à se donner pour objet la découverte de la Vérité, mais la découverte des jeux de mise en scène de la vérité comme 'croire' et 'faire croire"». Voir également, Boisson 2001, cité par Dendale et Coltier (2005: 127) : «En réalité, j'espère l'avoir montré par mon désossement analytique, la prise en charge, même si elle déborde la notion de vérité, implique nécessairement l'indication d'une valeur de vérité, concept décidément impossible à liquider facilement, quelque idée que l'on entretienne par ailleurs sur la nature de la vérité. »

5. Ce point trouve un écho dans l'entrée «Actions / événements (en narratologie)» du Dictionnaire d'Analyse du Discours (2002 : 26). La définition, rédigée par Jean-Michel Adam, s'y achève par cette conclusion : «Tout récit [...] peut être défini comme une interrogation portant 
sur les raisons d'agir, sur les degrés d'intentionnalité (motifs, buts) et donc sur la responsabilité des sujets ». Ce n'est pas le seul cas où les positions épistémologiques d'un chercheur en AD sont plus proches des théories de l'argumentation et d'une éthique du discours que la plupart de celles de leurs pairs. Jean-Michel Adam et Patrick Charaudeau accordent en effet une place centrale à l'analyse argumentative dans leurs travaux.

6. Je suis arrivé, affirme Eggs, «à une conclusion "contradictoire" mais simple; on ne peut pas réaliser l'ethos moral sans réaliser en même temps l'ethos neutre, objectif ou stratégique. Il faut agir et argumenter stratégiquement pour pouvoir réaliser la sobriété morale du débat. Ces deux faces de l'ethos constituent donc deux éléments de la même procédure : convaincre par le discours ».

7. Cette position est proche de celle défendue par Coltier et Dendale $(2004: 41 ; 44 ; 51)$ dans « La modalisation du discours de soi ». Qu'il s'agisse de "vérité pour le locuteur », de "vérité pour l'opinion publique » ou de vérité « onto-aléthique » de «l'ordre des choses », ce qui compterait en fait, c'est la description que le sujet donne de ce qu'il est et de ce qu'il «croit» ou «veut croire ".

8. J'emploierai désormais l'abréviation DictAD pour désigner cet ouvrage. V. également, l'entrée «Sujet du discours », p. 554-555.

9. V. les définitions de ce terme dans les entrées «Individuation» p.308-309 et « Positionnement » p. 453-454.

10. V. également à ce sujet Perelman $1989: 299$.

11. V. le DictAD (2002: 231) : «L'énonciation est fondamentalement prise dans l'interdiscours ». "L'énonciation revient à poser des frontières entre ce qui est "selectionné" et précisé peu à peu [...] et ce qui est rejeté. Ainsi se trouve dessiné en creux le champ de "tout ce à quoi s'oppose ce que le sujet a dit"».

12. Ceci ne signifie pas que l'argumentation soit une forme d'interaction verbale irénique et toujours consensuelle, mais que la conception du Vrai de celui qui remplit la fonction de l'Autre y a plus de poids que dans le cas de l'AD où elle serait réduite à n'exister qu' « en creux ».

13. Pour le recensement des effets d'objectivité analysés par Perelman et quant à son rapport à la question de l'objectivité discursive, cf. Koren (1993 : 469-487).

14. Il est intéressant de constater que Ducrot (2004:31) imagine l'existence dans les interactions verbales polémiques d'un tiers intériorisé par le sujet d'énonciation et son co-énonciateur, tiers à «apprivoiser» car il constituerait «cette sorte de sur-moi abstrait, que les interlocuteurs prennent comme arbitre idéal » et qui pourrait être la voix d'une doxa rationaliste objectiviste.

15. Cf.C. Kerbrat-Orecchioni 1981, "Argumentation et mauvaise foi".

16. Perelman ne cesse de répéter, affirme Kerbrat-Orecchioni (1981: 44) que «toute argumentation est contestable, et toute réfutation, à son tour réfutable »; il n'existerait donc pas de définition préalable absolue du «bon » et du «mauvais » argument; «bon » peut signifier «valable» ou "valide», tandis que «mauvais » peut renvoyer à «fautif» ou "abusif» " frauduleux », mais si le " caractère fluctuant de la norme argumentative » constitue un obstacle à la définition de la mauvaise foi, elle crée simultanément les conditions de possibilité qui permettent de penser son contraire : l'honnêteté discursive (cf. ibid. : 63).

17. Le Dictionnaire des Notions philosophiques considère l'intrication de la responsabilité juridique et de la responsabilité morale comme un trait distinctif de l'acception moderne du concept. On peut même y lire que si le sentiment de « responsabilité morale » n'était pas ressenti par le sujet et qu'il ne restait donc que la « responsabilité juridique », celle-ci ne serait qu'une «simple règle de jeu », « un peu d'habileté suffirait pour la tourner ou s'y soustraire» (p. 2251). L'effacement énonciatif pourrait donc être légitimement perçu, au prisme de cette remarque critique, comme une procédure langagière de refus d'assumer la responsabilité juridique de ses actes de parole mais cela n'annule pas pour autant la nécessité de penser l'autre versant de la responsabilité : le versant moral. 
18. C'est également à ce type de responsabilité envers la vérité référentielle que Searle (1982: 105) réfère dans sa théorie pragmatique des actes de langage. Ces actes doivent être accomplis «conformément à des règles » qui donnent lieu à des engagements (commitments). Ainsi l'assertion obéit à des « règles sémantiques et pragmatiques" tout à fait particulières dont la première et la quatrième sont les suivantes : 1. «La règle essentielle : l'auteur d'une assertion répond (commits himself to) de la vérité de la proposition exprimée »; 4 . «La règle de sincérité : le locuteur répond (commits himself to) de sa croyance dans la vérité de la proposition exprimée ». Searle (1972: 254) conclut: «"ignorer" l'emploi "engagé" des mots, aboutit en définitive à ignorer le langage lui-même ».

19. Dictionnaire des Notions philosophiques, "Subjectivité »(1990: 2479). Patrick Charaudeau (2002: 554), auteur de l'entrée "Sujet du discours", souligne que celui-ci est, "à la fois, surdéterminé-mais seulement en partie-par des conditionnements d'ordre divers, et libre d'opérer des choix lors de la mise en œuvre du discours. », entendu comme « libre de s'individuer, ce qui l'amène à user de stratégies".

20. Dictionnaire d'Analyse du Discours, «Sujet du discours » (2002:554).

21. Notions philosophiques, «Subjectivité » (1990: 2480).

22. C'est pour l'auditeur, affirme Oswald Ducrot (1972: 8), "une attitude considérée comme légitime que de se demander si le locuteur était autorisé à parler comme il l'a fait, et quelles intentions il pouvait avoir en le faisant. Les questions De quel droit dis-tu cela? ou Pourquoi dis-tu cela? passent pour des questions raisonnables ».

23. V., à ce sujet, Rhétoriques (1989 : 202-203, 430-431) et le Traité de l'argumentation (1970:679).

24. Rhétoriques (1989:431); et également, au sujet de la problématique de l'effacement énonciatif: le Traité (1970: 218, 243), Koren (1993: 475-479) et le Dictionnaire des Notions philosophiques (1990: 2252b), entrée « responsabilité », sur « faire le vide en soi ».

25. V. le Dictionnaire des Notions philosophiques (1990: 871b).

26. V. également le Dictionnaire des Notions philosophiques (1990:875b) ; et ci-dessous l'analyse de l'article du Monde intitulé «Évidences ».

27. Cf. Koren (2004). J'emprunte la notion d'« engagement neutre » à Charaudeau (1997: 262).

28. Il s'agit du premier et du dernier paragraphe de l'article, reproduits ici en entier ; l'exemple deux, par contre, ne cite que les trois premières lignes du troisième paragraphe. Les citations sélectionnées sont représentatives de la rhétorique à l'œuvre dans la totalité du texte. Je souhaite revisiter mon commentaire partiel de cet article, publié dans Semen 17, au prisme du concept d'éthique du discours.

29. V., au sujet de ce concept, Perelman et Olbrechts-Tyteca (1970: 42 et 75) : "Là où s'insère l'évidence rationnelle, l'adhésion de l'esprit semble suspendue à une vérité contraignante et les procédés d'argumentation ne jouent aucun rôle. L'individu, avec sa liberté de délibération et de choix, s'efface devant la raison qui le contraint et lui enlève toute possibilité de doute » $(42)$; « le sens commun » admet « l'existence de vérités indiscutées et indiscutables, il admet que certaines règles soient "hors discussion" et que certaines suggestions "ne méritent pas discussion". Un fait établi, une vérité évidente, une règle absolue, portent avec eux l'affirmation de leur caractère indiscutable, excluant la possibilité de défendre le pour et le contre ». La ressemblance avec la voix anonyme, mais péremptoire du locuteur du Monde est saisissante.

30. V., à propos des liens qui lient le concept d'argumentation à celui d'avenir, Perelman et Olbrechts-Tyteca (1970: 106-107).

31. Voir, à propos du concept de « récit » journalistique « bipolaire », Morin (1969: 155).

32. Voir Perelman et Olbrechts-Tyteca $(1970: 188)$ au sujet de la «manière statique » dont est abordée, hors du champ de l'argumentation, la signification essentiellement descriptive des notions.

33. Voir, à ce sujet, Koren $(1996: 259-267 ; 2001: 177-200 ; 2006 a)$. 
34. Voir, parmi tant d'autres, les deux exemples types suivants : cet extrait d'un texte de Simone de Beauvoir paru dans un article du Nouvel Observateur (12 janvier 1981) : «En disant tour à tour ou même simultanément blanc et noir, il [Le Monde] prétend donner leur chance à toutes les opinions : comme si le vrai et le faux s'équivalaient ", et cet énoncé relevé dans un reportage sur le terrorisme : «Trente années de terrorisme ou de résistance, comme on voudra »...

35. Une remarque critique épistémologique aurait donc le droit d'user de termes axiologiques ou axiologisés pour discréditer une position théorique rivale; il faudrait la distinguer de l'accusation formulée par les acteurs d'un échange polémique afin de discréditer leurs cibles. Un chercheur qui affirme ses réticences théoriques sur un ton radical serait toujours encore un scientifique « objectif »...

36. J'ai problématisé la question de la neutralité du chercheur linguiste dans Koren $(2002$; 2003 ; 2006 ; 2006a).

37. Il existe toutefois des exceptions ; ainsi les auteurs de "Discours en situation de travail ", Boutet, Gardin et Lacoste, paru en mars 1995 dans Langages 117, «Les analyses du discours en France», posent une question qui aurait pu constituer le point de départ d'un amendement de la doxa dominante : "Le linguiste peut-il dès lors se comporter uniquement en "linguiste" : faire abstraction de cette intrication du langagier et du non-langagier et traiter les situations de travail comme un lieu de recueil de corpus au même titre qu'un square ou un arrêt d'autobus? Pour deux ensembles de raisons nous pensons que non. D'abord pour des raisons éthiques. Ce que les femmes et les hommes au travail engagent d'énergie, de pulsions, de souffrance rendrait mal venue une attitude de linguiste consistant à venir chercher là des corpus exotiques. Cependant, même si nous n'avions pas de semblables dispositions éthiques, les propriétés mêmes de l'empirie observée nous contraindraient à répondre négativement. [...] Les collectifs se construisent, se transforment ou se détruisent sur le terrain par les actions langagières des participants, ils ne sont pas seulement des êtres de papier préconstitués ou créés par un discours monologal. Nous trouvons des agents engagés collectivement dans des activités et obligés de se servir du langage pour se coordonner, co-construire un objet, un processus, réaliser une tâche » (p. 16). Ce qui traduit parfaitement ici le « souci éthique» (p. 15) des trois chercheurs, c'est le fait de présenter leur position comme un choix et donc, « en creux ", les positions contraires non pas comme des positions « rejetées », mais comme des options tout aussi valides...

\section{RÉSUMÉS}

Cet article se situe à la croisée de l'analyse du discours, de la rhétorique argumentative et du concept philosophique d'éthique. Il a pour fin de problématiser la question du «positionnement» discursif. Je tenterai de réexaminer cette notion au prisme du concept argumentatif de « jugement » - et par « jugement » j'entends prise de position discursive - afin de contribuer à la conceptualisation de la notion d'éthique du discours. Cette démarche a pour fin de tenter de répondre à des questions épistémologiques comme : Pourquoi la prise de parole dans l'espace public implique-t-elle que le locuteur recoure, dans de nombreux genres de discours et même si les contraintes du genre ne l'exigent pas, à l'effacement énonciatif et donne à ses opinions les apparences du jugement de fait? Pourquoi est-il encore nécessaire aujourd'hui de rappeler que le lexique de la langue comprend des masses de termes subjectifs axiologiques et que des questions comme «Que pense X de cette question ? Est-il pour ou contre ? Est-ce bien ou mal à ses yeux? » constituent des réactions spontanées inéluctables aux dires des autres? 
Troisième et dernière question: Pourquoi un analyste du discours que préoccupe, certes, la question de la «véridiction", mais tout autant celle de la «rectitude éthique » et d'une rationalité axiologique, se trouve-t-il contraint de recourir à une théorie de l'argumentation pour pouvoir aborder les tenants et aboutissants de l'acte de juger hors de contextes polémiques aporétiques, alors que son objet de recherche est la mise en mots et la mise en scène discursives sous toutes leurs formes? Cet article commencera par l'élaboration d'un cadre théorique où seront traitées les questions suivantes : la nécessité de penser aux côtés de la prise en charge de la vérité référentielle et en interaction avec elle, la prise en charge des jugements de valeur et les conditions de possibilité d'une rationalité axiologique. Il faudra donc pouvoir penser la coexistence de la responsabilité discursive de l'énonciateur et de sa responsabilité argumentative, la question de la "véridiction » et celle de la « rectitude éthique ». Trois études de cas permettront ensuite de passer de la théorie à la pratique. Les deux premières concernent la rhétorique de l'écriture de presse. Il s'agit du concept déontologique d' " engagement neutre ») et d'un rythme fondamental de l'écriture de presse: l'oscillation statique entre deux pôles contraires. La troisième et dernière illustration concernera la controverse autour de la question du devoir de neutralité du chercheur en sciences du langage. La majorité des chercheurs prônent en effet un axiome déontologique de non-intervention; ils présupposent ainsi que la neutralité est linguistiquement et discursivement possible, alors qu'ils considèrent par ailleurs la subjectivité énonciative du langage comme un fait avéré.

This article is situated at the crossroads of Discourse Analysis, Argumentative Rhetoric and the philosophical concept of Ethics. It aims at raising the problems inherent to the issue of discursive positioning. It will attempt to reexamine this notion through the prism of the argumentative concept of judgment or discursive stand, in order to contribute to a conceptualization of the notion of an Ethics of discourse. This paper attempts to answer epistemological questions such as: 1) why does taking a stand in public imply that the speakers recur, in numerous genres of discourse, and even if the genre does not require it, to erasing any trace of discursive subjectivity, thus making opinions appear as judgments of fact? 2) Why is it still necessary today to remind linguists that the lexicon of a language contains a myriad of subjective axiological terms and that questions such as "what does X think about this? Is he for or against it? Does he like it or not?" constitute spontaneous inescapable reactions to the words of others? Lastly, why would a discourse analyst preoccupied by referential truth, but similarly by "ethical rectitude" and axiological rationality, have to turn to a theory of argumentation in order to deal with the ins and outs of judging? This article starts with a presentation of a theoretical frame dealing with the necessity to take into account not only referential truth, but also and in close relation to it, of value judgments and axiological rationality. The coexistence between the speaker's discursive responsibility and his argumentative responsibility, and the coexistence of the issues of "referential truth" and "ethical rectitude", should be considered jointly. Three case studies will then allow us to move from theory to practice. The first two cases deal with the rhetoric of the written press, analyzing first the deontological concept of "neutral commitment" and then a fundamental journalistic rhythm: the static oscillation between two opposing poles. The third and last example illustrates the controversial question of the linguist's neutrality. Most scholars subscribe to the deontological axiom of non-intervention, thus presupposing that neutrality is linguistically and discursively possible, although simultaneously affirming that enunciative subjectivity is inescapable. 
INDEX

Keywords : ethics of discourse, value judgment, axiological rationality, "objectivity" of the written press, researcher's commitment

Mots-clés : éthique du discours, prise en charge, jugement de valeur, rationalité axiologique, rhétorique « égalitaire » de la presse écrite, engagement du chercheur

\section{AUTEUR}

ROSELYNE KOREN

Université Bar-Ilan, ADARR 